\title{
Meropenem/Vaborbactam: A Review in Complicated Urinary Tract Infections
}

\author{
Sohita Dhillon ${ }^{1}$
}

Published online: 20 August 2018

๑) Springer Nature 2018, corrected publication August/2018

\begin{abstract}
The global threat of the spread of carbapenem resistance in Enterobacteriaceae has led to the search for new antibacterials. Intravenous meropenem/vaborbactam (Vabomere ${ }^{\mathrm{TM}}$ ) is the first carbapenem/ $\beta$-lactamase inhibitor combination approved in the USA for use in patients with complicated urinary tract infections (cUTIs), including pyelonephritis. Vaborbactam is a potent inhibitor of class A serine carbapenemases, which, when combined with the antibacterial meropenem, restores the activity of meropenem against $\beta$-lactamase producing Enterobacteriaceae, particularly Klebsiella pneumoniae carbapenemase (KPC)producing Enterobacteriaceae. Meropenem/vaborbactam demonstrated excellent in vitro activity against Gram-negative clinical isolates, including KPC- and extended-spectrum $\beta$-lactamase (ESBL)-producing Enterobacteriaceae. In the phase 3, noninferiority TANGO I trial in patients with cUTIs, intravenous meropenem/vaborbactam was noninferior to intravenous piperacillin/ tazobactam for overall success (composite of clinical cure and microbial eradication; FDA primary endpoint) and microbial eradication (EMA primary endpoint). In subsequent superiority testing, meropenem/vaborbactam was superior to piperacillin/tazobactam for overall success. Meropenem/vaborbactam was generally well tolerated, with a tolerability profile generally similar to that of piperacillin/tazobactam. TANGO I did not assess the efficacy of meropenem/vaborbactam for the treatment of infections caused by carbapenem-resistant Enterobacteriaceae and meropenem/vaborbactam is currently not indicated for these patients. Available evidence indicates that meropenem/vaborbactam is a useful treatment option for patients with cUTIs.
\end{abstract}

Meropenem/vaborbactam: clinical considerations in CUTI

First carbapenem/ $\beta$-lactamase inhibitor combination to be approved for use

Demonstrates excellent in vitro activity against Gramnegative clinical isolates, including KPC- and ESBLproducing Enterobacteriaceae

Noninferior to piperacillin/tazobactam in terms of clinical efficacy, and has generally similar tolerability

The manuscript was reviewed by: $D$. van Duin, Division of Infectious Diseases, University of North Carolina, Chapel Hill, NC, USA; J.L. Kuti, Hartford Hospital, Center for Anti-Infective Research and Development, Hartford, CT, USA; H.S. Sader, JMI Laboratories, North Liberty, IA, USA.

Sohita Dhillon

demail@springer.com

1 Springer, Private Bag 65901, Mairangi Bay, Auckland 0754, New Zealand

\section{Introduction}

Complicated urinary tract infections (cUTIs) place a heavy burden on healthcare systems and are a common cause of hospitalization [1]. They are caused by a broad range of bacteria, with Enterobacteriaceae (e.g. Escherichia coli) being the predominant pathogen $[2,3]$. Patients with cUTIs are usually treated with antibacterials, such as cephalosporins, fluoroquinolones, aminoglycosides and carbapenems (e.g. doripenem, ertapenem) $[4,5]$. Carbapenems are highly potent, broad-spectrum $\beta$-lactam antibacterial agents, with a unique structure that confers protection against most $\beta$-lactamases [e.g. metallo- $\beta$-lactamase (MBL) and extended-spectrum $\beta$-lactamase (ESBL)] [6, 7], and are generally used as first-line empirical therapy for cUTIs [8]. However, the emergence and spread of carbapenem resistance in Enterobacteriaceae since the 1990s poses a growing threat to public health worldwide $[9,10]$. The US Centers for Disease Control and Prevention have identified carbapenemresistant Enterobacteriaceae as an urgent threat [11] and the development of new antibiotics against these bacteria has been recognized as a critical need by the WHO [12]. 
There are several mechanisms of resistance to carbapenems, including enzymatic inactivation, target-site mutation, porin mutations and efflux pumps [6]. Production of carbapenemases that can hydrolyse most $\beta$-lactams, including carbapenems [13], is one of the main mechanisms of resistance [14], with Klebsiella pneumoniae carbapenemase (KPC) being the most common carbapenemase in the USA [13]. Restoring the activity of $\beta$-lactams by combining them with $\beta$-lactamase inhibitors (BLIs) has been an effective strategy to overcome $\beta$-lactamase-mediated resistance to $\beta$-lactams, with ceftazidime/avibactam and ceftolozane/tazobactam combinations currently approved for use in patients with cUTIs $[15,16]$. However, KPC is poorly inhibited by older BLIs (e.g. clavulanic acid and tazobactam); this led to the development of vaborbactam, a first-in-class boronic acid transitional state inhibitor (BATSI) that potently inhibits class A carbapenemases (particularly KPC), thereby restoring the activity of carbapenems against KPC-producing Enterobacteriaceae [15, 16]. Meropenem/vaborbactam (Vabomere $^{\mathrm{TM}}$ ) is the first carbapenem/BLI combination approved in the USA for use in patients with cUTI, including pyelonephritis [17]. This article reviews the efficacy and tolerability of intravenous meropenem/vaborbactam in this indication and overviews its pharmacological properties.

\section{Pharmacodynamic Properties of Meropenem/Vaborbactam}

\subsection{Mechanism of Action}

Meropenem, a carbapenem antibacterial agent, is stable to hydrolysis by most beta-lactamases produced by Gram-negative and Gram-positive bacteria, including penicillinases and cephalosporinases [17]. Meropenem exerts its bactericidal action by binding to penicillin-binding proteins (PBPs) in the bacterial cell wall and inhibiting peptidoglycan crosslinking associated with cell wall synthesis, which ultimately leads to cell death [17-19].

Vaborbactam is a non-suicidal, broad-spectrum BATSI designed as a potent inhibitor of class A serine carbapenemases, such as KPC, NMC-A and SME-2, as well as other class A (e.g. CTX-M, SHV) and class C (e.g. P99, MIR) $\beta$-lactamases [15-17]. Vaborbactam was also shown to inhibit the newly discovered class A carbapenemases BKC-1 and FRI-1 found in clinical isolates of K. pneumoniae and Enterobacter cloacae, respectively [20]. Class B (e.g. NDM, VIM) and class D (e.g. OXA-48) carbapenemases are not inhibited by vaborbactam [16]. Vaborbactam does not inhibit mammalian serine proteases [15] and does not have any antibacterial activity $[17,21]$.
Vaborbactam protects meropenem from degradation by serine carbapenemases via a novel mechanism of enzyme inhibition. This involves the formation of a covalent adduct between the catalytic serine residue of $\beta$-lactamases and the boron moiety of vaborbactam, which mimics the tetrahedral transition state of $\beta$-lactamase hydrolysis [15, $18,22]$. Biochemical studies showed that vaborbactam is a slow, tight-binding reversible inhibitor of KPC-2, with a very slow off-rate (enzyme residence time of $992 \mathrm{~min}$ ) and $1: 1$ stoichiometry $[22,23]$. It was suggested that the slow off-rate of vaborbactam for KPC may play an important role in enhancing the activity of antibacterials against KPC-producing bacteria [16]. Vaborbactam inhibited KPC-2 mediated hydrolysis of nitrocefin with greater potency than tazobactam (> 20-fold) and clavulanic acid (> 500-fold) [15, 23]. Unlike other currently available BLIs (such as tazobactam and avibactam), vaborbactam is not hydrolyzed by KPC [22].

In vitro, meropenem combined with vaborbactam reduced the minimum inhibitory concentration (MIC) values of meropenem by $\geq 64$-fold against engineered strains and clinical isolates producing class A serine carbapenemases (e.g. KPC-2, KPC-3, SME-2, NMC-A) [15, 16], including strains coproducing KPC and class A ESBLs (e.g. TEM-1 and SHV-1) [15] or KPC-producing strains carrying derepressed $A m p C$ genes [21]. Vaborbactam also restored the potency of meropenem against engineered KPC-producing K. pneumoniae strains with increased MICs because of porin mutations [16, 24]. Like carbapenems, vaborbactam crosses the outer membrane using both the OmpK35 and OmpK36 porins, but unlike carbapenems that can use both porins with similar efficiency, OmpK36 appears to be the preferred porin for vaborbactam [16]. Vaborbactam, unlike meropenem, is not a substrate of the multidrug resistance (MDR) efflux pump AcrAB-TolC [16].

\subsection{Antibacterial Activity}

\subsubsection{In Vitro and In Vivo Activity}

This section focuses on the antibacterial activity of meropenem/vaborbactam against Gram-negative K. pneumoniae, $E$. coli and $E$. cloacae species complex microorganisms associated with cUTI (i.e. pathogens against which meropenem/ vaborbactam demonstrated efficacy in vitro and in clinical infections) [17]. Meropenem/vaborbactam has also shown good in vitro activity against isolates of a similar genus or organism group (e.g. Citrobacter freundii, Citrobacter koseri, Enterobacter aerogenes, Klebsiella oxytoca), with at least $90 \%$ of bacteria exhibiting an in vitro $\mathrm{MIC}\left(\mathrm{MIC}_{90}\right)$ of less than or equal to the US Food and Drug Administration (FDA) proposed susceptibility breakpoint against Enterobacteriaceae ( $\leq 4 / 8 \mu \mathrm{g} / \mathrm{mL}$; assessed by broth microdilution). 
However, its efficacy in treating clinical infections caused by these bacteria remains to be established in adequate, wellcontrolled clinical trials [17].

Meropenem/vaborbactam demonstrated excellent in vitro activity against Gram-negative clinical isolates, including KPC- and ESBL-producing Enterobacteriaceae and MDR Enterobacteriaceae (Table 1). Where reported, isolates were collected from bloodstream infections [25-28], pneumonia in hospitalized patients [25, 26, 28], skin/skin structure/soft tissue infections [25, 26, 28], UTIs [25, 26, 28, 29], intraabdominal infections $[25,26,28]$ and other less prevalent or undetermined clinical specimen types [25, 26, 28].

Against contemporary Enterobacteriaceae, K. pneumoniae, E. coli and/or E. cloacae isolates collected worldwide, meropenem/vaborbactam showed potent in vitro activity that was greater than that of piperacillin/tazobactam (Table 1) [25-31]. The in vitro activity of meropenem/vaborbactam against Enterobacteriaceae, K. pneumoniae and E. coli isolates was also greater than that of cefepime and ceftazidime. For example, against Enterobacteriaceae, the $\mathrm{MIC}_{90}$ values for meropenem/vaborbactam were $0.03-0.06 \mu \mathrm{g} / \mathrm{mL}$ versus $4-16 \mu \mathrm{g} / \mathrm{mL}$ for cefepime and $8-32 \mu \mathrm{g} / \mathrm{mL}$ for ceftazidime, and the susceptibility rates were $98.7-100 \%$ versus $83.9-89.6 \%$ (CLSI breakpoint)/83.2-88.4\% (EUCAST) and $83.1-88.2 \%$ (CLSI)/79.2-85.0\% (EUCAST) [25-31]. The $\mathrm{MIC}_{90}$ of meropenem/vaborbactam compared with tigecycline was $0.03-0.06$ versus $0.5-1.0 \mu \mathrm{g} / \mathrm{mL}$, and the susceptibility rates were $98.7-100 \%$ versus $98.0-99.5 \%$ (FDA)/92.6-92.2\% (EUCAST) [25-29]. In the pivotal TANGO I trial (Sect. 4), the in vitro activity of meropenem/ vaborbactam against Gram-negative clinical isolates from urine at baseline was consistent with that of in vitro studies, with $\mathrm{MIC}_{90}$ values of $\leq 0.06 \mu \mathrm{g} / \mathrm{mL}$ for all Enterobacteriaceae compared with $\leq 0.5 \mu \mathrm{g} / \mathrm{mL}$ for meropenem, $>64 \mu \mathrm{g} /$ $\mathrm{mL}$ for piperacillin/tazobactam and cefepime, and $2 \mu \mathrm{g} / \mathrm{mL}$ for tigecycline [32]. These isolates produced one or more of the following $\beta$-lactamase enzymes: KPC, CTX-M,TEM, SHV, CMY, ACT and OXA (non-carbapenemases) [17].

Meropenem/vaborbactam had greater activity against its main targets, KPC-producing Enterobacteriaceae, $K$. pneumoniae, E. coli and E. cloacae, than several other antibacterials, including meropenem, piperacillin/tazobactam, cefepime and ceftazidime. For example, against KPC-producing Enterobacteriaceae, the susceptibility rates for meropenem/vaborbactam (99.0-99.3\%; Table 1) were greater than the rates for meropenem $(\leq 5.2 \%$; Table 1$)$, piperacillin/tazobactam $(0.7 \%$; Table 1$)$, cefepime $[1.5 \%$ (CLSI and EUCAST)] and ceftazidime [1.5\% (CLSI)/0.7 (EUCAST)] [25, 30, 33, 34]. The in vitro activity of meropenem/vaborbactam against KPC-producing Enterobacteriaceae was generally similar to that of tigecycline and the $\beta$-lactam BLI combination ceftazidime/avibactam, with susceptibility rates of $99.0-99.3 \%$ for meropenem/vaborbactam versus $95.8-100 \%$ (FDA)/96.3\% (EUCAST) for tigecycline and $98.2 \%$ (FDA) for ceftazidime/avibactam [25, 33].

Meropenem/vaborbactam had generally similar in vitro activity against KPC-2- and KPC-3-producing Enterobacteriaceae isolates, with $\mathrm{MIC}_{90}$ values of $1 \mu \mathrm{g} / \mathrm{mL}$ (both carbapenemases) and susceptibility rates of 99.2 and $98.7 \%$, respectively (FDA breakpoint) [33]. There was also no appreciable difference in the in vitro activity of meropenem/ vaborbactam against Enterobacteriaceae isolates coproducing KPC and ESBLs and/or AmpC enzymes, and those producing only KPC (e.g. $\mathrm{MIC}_{90} 0.06 \mu \mathrm{g} / \mathrm{mL}$ for KPC AmpC and $1.0 \mu \mathrm{g} / \mathrm{mL}$ for KPC ESBL vs. $1.0 \mu \mathrm{g} / \mathrm{mL}$ for KPC isolates) [33].

Carbapenem-resistant Enterobacteriaceae (CRE) are usually resistant to most available $\beta$-lactams, including carbapenems, penicillins, cephalosporins and their combinations with BLIs [35]. Of some selected antibacterials, meropenem/vaborbactam and tigecycline exhibited very good in vitro antibacterial activity against CRE isolates [25-29, 31], with susceptibility rates for meropenem/vaborbactam (66.2-100\%; Table 1) and tigecycline [99.1-100\% (FDA)/86.1-92.5\% (EUCAST)] higher than those for meropenem $(\leq 10.4 \%$; Table 1$)$, piperacillin/tazobactam $(\leq 6.7 \%$; Table 1), cefepime [3.6-7.2\% (CLSI)/1.0-6.7\% (EUCAST)] and ceftazidime $(0-6.0 \%$ (CLSI) $/ 0-5.7 \%$ (EUCAST)] [25-29, 31].

Meropenem/vaborbactam also had excellent in vitro antibacterial activity against ESBL-producing Enterobacteriaceae, E. coli and K. pneumoniae (100\% susceptibility; Table 1). Meropenem/vaborbactam in vitro activity against ESBL-producing Enterobacteriaceae was generally similar to that of tigecycline [susceptibility rates 100 vs. 100 (FDA)/94.9\% (EUCAST)] and numerically greater than that of meropenem, piperacillin/tazobactam and ceftazidime [100 vs. $83.8 / 85.9 \%, 23.2 / 19.2 \%$ and $19.2 / 17.1 \%$, respectively (CLSI/EUCAST)] [27]. In the TANGO I clinical trial, meropenem/vaborbactam had the greatest in vitro activity against ESBL-producing Enterobacteriaceae, $K$. pneumoniae, E. coli and E. cloacae. For example, against Enterobacteriaceae, the $\mathrm{MIC}_{90}$ for meropenem/vaborbactam was $0.125 \mu \mathrm{g} / \mathrm{mL}$ compared with $\leq 0.5 \mu \mathrm{g} / \mathrm{mL}$ for meropenem, $>64 \mu \mathrm{g} / \mathrm{mL}$ for piperacillin/tazobactam, $2 \mu \mathrm{g} /$ $\mathrm{mL}$ for tigecycline and $>64 \mu \mathrm{g} / \mathrm{mL}$ for ceftazidime [32].

Meropenem/vaborbactam exhibited more potent in vitro activity against MDR Enterobacteriaceae isolates than meropenem, piperacillin/tazobactam, cefepime and ceftazidime ( $\mathrm{MIC}_{90}$ values $>16$-fold lower than the comparators), while its activity against XDR Enterobacteriaceae isolates was generally similar to that of the comparators $\left(\mathrm{MIC}_{90} 32\right.$ vs. $>16$ to $>64 \mu \mathrm{g} / \mathrm{mL}$ ) [25]. Tigecycline exhibited potent in vitro activity against both MDR and XDR Enterobacteriaceae, with $\mathrm{MIC}_{90}$ values of 1 and $2 \mu \mathrm{g} / \mathrm{mL}$, respectively [25]. 
Table 1 Comparative in vitro activity of meropenem/vaborbactam against Gram-negative clinical isolates

\begin{tabular}{|c|c|c|c|c|c|c|c|}
\hline Pathogen & No. of isolates ${ }^{\mathrm{a}}$ & $\begin{array}{l}\operatorname{MEV~MIC}{ }_{90}{ }^{a} \\
\mu \mathrm{g} / \mathrm{mL}\end{array}$ & $\begin{array}{l}\text { MEV } \% S^{a, b} \\
\text { US FDA }\end{array}$ & $\begin{array}{l}\text { MEM MIC }{ }_{90}{ }^{a} \\
\mu \mathrm{g} / \mathrm{mL}\end{array}$ & $\begin{array}{l}\text { MEM \% } \mathrm{S}^{\mathrm{a}, \mathrm{c}} \\
\mathrm{CLSI} / \mathrm{EUCAST}\end{array}$ & $\begin{array}{l}\mathrm{TZP} \mathrm{MIC}_{90}{ }^{\mathrm{a}} \\
\mu \mathrm{g} / \mathrm{ml}\end{array}$ & $\begin{array}{l}\text { TZP } \% S^{\mathrm{a}, \mathrm{c}} \\
\text { CLSI/EUCAST }\end{array}$ \\
\hline Enterobacteriaceae [25-31] & 46,769 & 0.03 to 0.06 & $98.7-100$ & 0.06 & 96.6-98.7/96.9-98.4 & $8-16$ & $92.0-93.2 / 88.8-89.0$ \\
\hline Klebsiella pneumonia [25-29] & 5876 & $0.03-0.12$ & $97.0-100$ & $0.03-0.12$ & $88.3-94.3 / 93.0-95.0$ & 32 to $>64$ & $87.8-88.2 / 80.7-82.4$ \\
\hline Escherichia coli [25-29] & 11,514 & $\begin{array}{l}\leq 0.015 \text { to } \\
0.03\end{array}$ & $99.8-100$ & 0.03 & $99.7-99.8 / 99.7$ & 8 & $94.7-95.7 / 91.2-93.3$ \\
\hline Enterobacter cloacae spp. [25-28] & 2572 & 0.03 & $99.8-100$ & & 97.2/NR & & \\
\hline CRE $[25-29,31]$ & 1003 & $0.5-32^{\mathrm{d}}$ & $66.2-100^{\mathrm{e}}$ & $>32$ & $0-3.1 / 6.0-10.4$ & $>64$ to $>128$ & $0-3.0 / 0-6.7$ \\
\hline Serine-CPE [21] & 315 & 1 & 97.8 & $>64$ & $2.2 / 7.3$ & & \\
\hline MDR [25] & 1210 & 1 & & 32 & $77.7 / 80.2$ & $>64$ & $36.6 / 28.7$ \\
\hline XDR [25] & 161 & 32 & & $>32$ & $13.0 / 19.9$ & $>64$ & $2.5 / 2.5$ \\
\hline \multicolumn{8}{|l|}{ ESBL-phenotype } \\
\hline Enterobacteriaceae [27] & 99 & 0.12 & 100 & 16 & $83.8 / 85.9$ & $>64$ & $65.7 / 50.5$ \\
\hline Klebsiella pneumonia [27] & 33 & 0.5 & 100 & & & & \\
\hline E. coli $[27,29]$ & 148 & 0.03 & 100 & 0.06 & 83.7-96.5/NR & 32 & $83.7 / \mathrm{NR}$ \\
\hline \multicolumn{8}{|l|}{ KPC-producing } \\
\hline Enterobacteriaceae $[25,30,33,34]$ & 1404 & $0.5-8$ & $99.0-99.3$ & $>32$ to $>64$ & $0-0.7 / 3.4-5.2$ & $>64$ & $0.7 / 0.7$ \\
\hline CRE [28] & 206 & 1 & 99.5 & $>32$ & $1.9 / 7.3$ & & \\
\hline K. pneumonia $[21,24,33]$ & 1207 & $0.5-1$ & $96.6-98.9$ & $>32$ to $>64$ & $0-7.0 / 2.4$ & & \\
\hline E. coli $[21,33]$ & 56 & $\begin{array}{l}\leq 0.03 \\
\text { to } \leq 0.06\end{array}$ & 100 & $8-16$ & $0-19.1 / 38.1$ & & \\
\hline $\begin{array}{l}\text { E. cloacae [21] or Enterobacter spp. } \\
{[33]^{\mathrm{f}}}\end{array}$ & & $0.12-0.25$ & 100 & $\geq 32$ & $0-2.6 / 10.3$ & & \\
\hline $\begin{array}{l}\text { non-KPC-producing CRE } \\
{[25,28]}\end{array}$ & 250 & $>32$ & 31.4 & $>32$ & $1.7-3.1 / 1.7-5.0$ & $>64$ & $5.4 / 4.7$ \\
\hline CNE [25] & 63 & 4 & & 16 & $3.2 / 7.9$ & $>64$ & $9.5 / 7.9$ \\
\hline MBL-producing CRE ${ }^{\mathrm{g}}[28,30]$ & 111 & $>32$ & $3.8-18.6$ & $>32$ & $0-8.5 / 0-1.7$ & & \\
\hline
\end{tabular}

Assessed using CLSI broth microdilution methods; agar dilution method used for comparators in one study [24]. Clinical isolates were collected in the USA (2014 [27, 29], 2015 [26]), Europe (2014-2015 [30]), New York city (2013-2014 [24]), China (2006-2012 [34]) and worldwide (2000-2013 [21], 2014 [25], 2014-2015 [33], 2015 [28]). Some data available only as abstract and/or posters [26, 27, 29, 30, 34]

$C L S I$ Clinical and Laboratory Standards Institute, CNE carbapenemase-negative Enterobacteriaceae, CPE carbapenemase-producing Enterobacteriaceae, $C R E$ carbapenem-resistant Enterobacteriaceae, ESBL Extended-Spectrum $\beta$-Lactamase, EUCAST European Committee on Antimicrobial Susceptibility Testing, KPC Klebsiella pneumoniae carbapenemase, $M D R$ multidrug resistant, $M E M$ meropenem, $M E V$ meropenem/ vaborbactam, $M I C_{90}$ minimum inhibitory concentration required to inhibit $90 \%$ of isolates, $N R$ not reported, $S$ susceptible, $T Z P$ piperacillin/ tazobactam, $X D R$ extensively drug resistant, $M B L$ metallo- $\beta$-lactamase

${ }^{a}$ Across all studies; MIC $_{90}$ and susceptibility not reported for all studies

${ }^{\mathrm{b}}$ Susceptibility estimated based on US FDA susceptibility interpretive criteria

${ }^{\mathrm{c}}$ According to CLSI or EUCAST breakpoints

${ }^{\mathrm{d}} \mathrm{MIC}_{90}$ (no. of isolates): 0.5-2.0 $\mu \mathrm{g} / \mathrm{mL}(n=15-96)$ [26, 27, 29], $32 \mu \mathrm{g} / \mathrm{mL}(n=265$ [25] and 330 [28]) and NR $(n=281)$ [31]

e'Susceptibility (no. of isolates): 95.8-100\% ( $n=15-96)$ [26, 27, 29], 73.9\% $(n=330)$ [28], 66.2\% $(n=281)$ [31] and NR $(n=265)$ [25]

${ }^{\mathrm{f}}$ Including 17 E. cloacae isolates

${ }^{g}$ Including 49 NDM, one IMP-64 and two VIM-producers [28], and 41 NDM-1 and 18 VIM-like producers [30]

Against $P$. aeruginosa isolates ( $n=2640)$, meropenem/ vaborbactam demonstrated generally similar in vitro activity to that of meropenem and piperacillin/tazobactam, with $\mathrm{MIC}_{90}$ values of $8-32 \mu \mathrm{g} / \mathrm{mL}$ for meropenem/vaborbactam and meropenem, and $>64$ to $>128 \mu \mathrm{g} / \mathrm{mL}$ for piperacillin/ tazobactam, with susceptibility rates of 86.3 (FDA), 78.4\% (CLSI and EUCAST) and 79.2\% (CLSI and EUCAST), respectively [25].

The in vitro activity of meropenem/vaborbactam was supported by evidence from animal models of infections due to carbapenem-resistant $K$. pneumoniae, E. cloacae and E. coli isolates [meropenem/vaborbactam MICs $\leq 0.06$ to $16 \mu \mathrm{g} / \mathrm{mL}$ (with vaborbactam $8 \mu \mathrm{g} / \mathrm{mL}$ )] [22, 36, 37], including murine models of urinary tract infection $[22,36]$ or neutropenic thigh or lung infections [37]. At human-simulated exposures of meropenem plus vaborbactam (i.e. $2 \mathrm{~g}$ of each component every $8 \mathrm{~h}$ by 3 -h infusion), bacterial killing against all strains tested was 0.8-2.89 logs colony forming units (CFU)/tissue $[36,37]$. 


\subsubsection{Resistance}

Resistance to $\beta$-lactam antibacterials involves several mechanisms, including the production of $\beta$-lactamases, modification of PBPs by gene acquisition or target alteration, upregulation of efflux pumps and loss of outer membrane porin [17]. Clinical isolates may produce multiple $\beta$-lactamases, express varying levels of $\beta$-lactamases or have amino acid sequence variations, as well as other resistance mechanisms that remain to be identified [17].

In vitro studies showed that meropenem/vaborbactam may have reduced potency against clinical isolates coproducing KPC and class B or D carbapenemases, or those with porin mutations and overexpression of efflux pumps [17]. In one global study, seven (all K. pneumoniae) of 315 serine-carbapenemase-producing Enterobacteriaceae had $\mathrm{MIC}_{90}$ values of $\geq 16 \mu \mathrm{g} / \mathrm{mL}$, and of these, four isolates coproduced KPC enzymes with an MBL [VIM-1 and VIM-4 one and three isolate(s), respectively] [21]. The remaining three isolates with elevated $\mathrm{MIC}_{90}$ values had reduced expression of ompK37 and modest-to-high expression (6.62-22.3-fold) of the AcrAB-TolC resistance modulation efflux system [21]. In another surveillance study, 511 of 517 (98.8\%) CRE isolates harbouring $b l a_{K P C}$ were susceptible to meropenem/vaborbactam at the FDA breakpoint of $\leq 4 / 8 \mu \mathrm{g} /$ $\mathrm{mL}$; three isolates with elevated meropenem/vaborbactam MIC values of $>8 \mu \mathrm{g} / \mathrm{mL}$ coharboured bla $_{K P C}$ with $b l a_{\mathrm{NDM}-1}$ or bla $a_{\text {OXA-48 }}$-like, or had a nonsense mutation in ompK35 [38]. Of note, bla ${ }_{K P C}$ carrying isolates were not susceptible to meropenem, imipenem or doripenem $\left(\mathrm{MIC}_{90}\right.$ values of $>32,>8$ and $>4 \mu \mathrm{g} / \mathrm{mL}$, respectively) and were largely (92.2-99.6\%) resistant to cephalosporins and piperacillin/ tazobactam (CLSI breakpoints) [38]. In a third study, only one of 206 KPC-producing Enterobacteriaceae isolates had an $\mathrm{MIC}_{90}$ value $>4 / 8 \mu \mathrm{g} / \mathrm{mL}$, and sequence analysis of the outer membrane proteins of this isolate revealed a frame shift in OmpK35 (which is likely to be incompatible with function) and insertion of 134GD in loop 3 of OmpK36 [28].

Ceftazidime/avibactam has been associated with treatment-emergent resistance [39-41], with mechanisms including genomic adaptations in $b l a_{K P C-2}$ [39] and mutations in

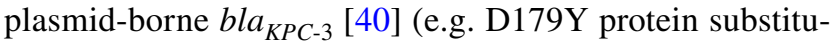
tion). Vaborbactam is not affected by KPC-2 and KPC-3 variants containing D179Y amino acid substitution, which results in increased catalytic efficiency for ceftazidime hydrolysis and resistance to avibactam [42]. The S130 residue, which plays an important role in the catalytic mechanism of $\beta$-lactamases and a significant role in the inhibition of KPC-2 by other BLIs (e.g. avibactam), also does not appear to be involved in the inhibition of KPC-2 by vaborbactam [43]. In a study of 991 KPC-producing, OXA-48 and MBL negative Enterobacteriaceae isolates, 14 of 18 ceftazidime/avibactam-resistant isolates $\left(\mathrm{MIC}_{90} \geq 16 \mu \mathrm{g} / \mathrm{mL}\right)$ were susceptible to meropenem/vaborbactam $\left(\mathrm{MIC}_{90} \leq 4 \mu \mathrm{g} / \mathrm{mL}\right)$ and 6 of 10 meropenem/vaborbactam nonsusceptible isolates $\left(\mathrm{MIC}_{90} \geq 8 \mu \mathrm{g} / \mathrm{mL}\right.$ ) were susceptible to ceftazidime/avibac$\operatorname{tam}\left(\mathrm{MIC}_{90} \leq 8 \mu \mathrm{g} / \mathrm{mL}\right)$ [33]. Cross resistance between meropenem/vaborbactam and ceftazidime/avibactam occurred in $20.8 \%$ (5 of 24) of isolates resistant to either agent [33].

An in vitro study determined meropenem and vaborbactam concentrations associated with selecting or preventing single-step mutations in 18 KPC-producing $K$. pneumoniae strains from global surveillance studies (meropenem MIC 8-512 $\mu \mathrm{g} / \mathrm{mL}$ and meropenem/vaborbactam $\mathrm{MIC} \leq 0.06-32 \mu \mathrm{g} / \mathrm{mL}$ ), with an emphasis on strains in the upper range of meropenem/vaborbactam MIC distribution [44]. With meropenem and vaborbactam at $8 \mu \mathrm{g} /$ $\mathrm{mL}$ each, the drug-resistance mutation frequency was suppressed to $<1 \times 10^{-8}$ in $77.8 \%$ (14 of 18 ) of these strains (all of which had MICs $\leq 8 \mu \mathrm{g} / \mathrm{mL}$ ), and with meropenem $16 \mu \mathrm{g}$ / $\mathrm{mL}$ plus vaborbactam $8 \mu \mathrm{g} / \mathrm{mL}$ all 18 strains were inhibited, including the most resistant strains (MICs $16-32 \mu \mathrm{g} / \mathrm{mL}$ ) [44].

In an in vitro hollow-fibre infection model, drug exposures similar to meropenem $2 \mathrm{~g}$ with vaborbactam $2 \mathrm{~g}$ administered every $8 \mathrm{~h}$ by 3 -h infusion in patients in phase 3 studies produced $\approx 6 \log$ s of bacterial killing in three tested KPC-producing $K$. pneumoniae isolates with multiple mechanisms of carbapenem resistance and meropenem/vaborbactam MICs of $8-16 \mu \mathrm{g} / \mathrm{mL}$ (with vaborbactam $8 \mu \mathrm{g} / \mathrm{mL}$ ) [45]. This exposure dose also suppressed the development of resistance, with no changes in MIC between pre- and post-treatment isolates, suggesting that this dosage regimen will be effective against Enterobacteriaceae with MICs of $8-16 \mu \mathrm{g} / \mathrm{mL}$, and supporting its use in phase 3 studies [45].

\subsubsection{Pharmacodynamic/Pharmacokinetic Considerations}

Like other $\beta$-lactam antibacterials, the best correlate with efficacy of meropenem/vaborbactam is the percentage of time of a dosing interval that the unbound plasma concentration of meropenem exceeds the meropenem/vaborbactam MIC (\% $\mathrm{T}$ > MIC) against the infecting organism [17]. In neutropenic mouse models of infection, the magnitude of meropenem \% $\mathrm{T}>\mathrm{MIC}$ associated with bacterial stasis and a 1 - and $2-\log _{10}$ CFU reduction from baseline was determined to be 30,35 and $45 \%$, respectively, for Gram-negative bacilli [46, 47]. The pharmacokinetic-pharmacodynamic (PK-PD) parameter that best described the antibacterial activity of vaborbactam in combination with meropenem at exposures equivalent to $2 \mathrm{~g}$ every $8 \mathrm{~h}$ by 3 -h infusion in humans was the ratio of the 24-h unbound plasma vaborbactam area under the concentration time curve (AUC) to meropenem/vaborbactam MIC ( $f$ AUC:MIC), according to data from neutropenic mouse thigh and in vitro hollow-fibre infection models $[17,48]$. In the neutropenic mouse model 
of thigh infection, a 24-h fAUC:MIC of 38 was associated with restoring the bacterial killing effect of meropenem to $1-\log _{10}$ CFU reduction from baseline in KPC-producing strains, and was considered as the vaborbactam PK-PD target by the FDA [46].

PK-PD target attainment analyses (using Monte Carlo simulation, population pharmacokinetic models, non-clinical PK-PD targets for efficacy and in vitro surveillance data) evaluated the meropenem/vaborbactam dosage regimens selected for patients with normal renal function or varying degrees of renal impairment [estimated glomerular filtration rates (eGFRs) $\geq 50,30-49,15-29$ or $<15 \mathrm{~mL} / \mathrm{min} / 1.73 \mathrm{~m}^{2}$ receiving $2 / 2,1 / 1,1 / 1$ or $0.5 / 0.5 \mathrm{~g}$ of each component, respectively, every $8 \mathrm{~h}$ by 3 -h infusion] [47]. Based on the three meropenem $\% f \mathrm{~T}>\mathrm{MIC}$ targets (i.e. 30, 35 and $45 \%$ ) and pathogen MIC distributions, the percentage probabilities of PK-PD target attainment at meropenem/vaborbactam MICs of 4 and $8 \mu \mathrm{g} / \mathrm{mL}$ were $\geq 98.5$ and $\geq 90.9 \%$, respectively [47]. The $f$ AUC:MIC of vaborbactam achieved with a regimen of meropenem $2 \mathrm{~g}$ and vaborbactam $2 \mathrm{~g}$ every $8 \mathrm{~h}$ by 3 -h infusion in patients with KPC-producing Enterobacteriaceae at baseline was $\geq 50$-fold higher than the $f$ AUC:MIC target of 38 [46]. These results support the use of the approved dosage regimen of meropenem/vaborbactam $4 \mathrm{~g}$ (meropenem $2 \mathrm{~g}$ and vaborbactam $2 \mathrm{~g}$ ) every $8 \mathrm{~h}$ by $3-\mathrm{h}$ infusion, as well as dosage adjustments for impaired renal function (Sect. 6).

\section{Pharmacokinetic Properties of Meropenem/Vaborbactam}

The pharmacokinetic properties of meropenem and vaborbactam following administration of meropenem/vaborbactam $4 \mathrm{~g}(2 / 2 \mathrm{~g})$ every $8 \mathrm{~h}$ as 3 -h intravenous infusion in healthy adults [17] and in a population pharmacokinetic analysis in patients with cUTIs or other infections due to CRE [17, 49] are summarized in Table 2.

Meropenem and vaborbactam peak plasma concentrations and AUC increased dose proportionally over a dose range of meropenem $1-2 \mathrm{~g}$ and vaborbactam $0.25-2 \mathrm{~g}$ when administered as a 3-h intravenous infusion [17]. There was no accumulation of meropenem/vaborbactam in healthy adults after multiple 3-h infusions every $8 \mathrm{~h}$ for 7 days [17]. In infected patients, the steady-state volumes of distribution of meropenem and vaborbactam were 20.2 and $18.6 \mathrm{~L}$, respectively, in a population pharmacokinetic analysis $[4,17]$. Approximately $2 \%$ of meropenem and $33 \%$ of vaborbactam is plasma protein bound [17]. Hydrolysis of the $\beta$-lactam ring to inactive meropenem open lactam is a minor metabolic pathway, accounting for $22 \%$ of a meropenem dose eliminated in the urine $[4,17]$. Vaborbactam is not metabolized. Meropenem and vaborbactam are largely eliminated by the kidneys, with
$40-60 \%$ of a meropenem dose and $75-90 \%$ of a vaborbactam dose eliminated as unchanged drug within 24 to $48 \mathrm{~h}$. The mean renal and non-renal clearance of meropenem was 7.8 and $7.3 \mathrm{~L} / \mathrm{h}$, respectively; corresponding values for vaborbactam were 8.9 and $2.0 \mathrm{~L} / \mathrm{h}$ [17].

Hepatic impairment does not affect the pharmacokinetic properties of meropenem, and vaborbactam does not undergo hepatic metabolism; thus, hepatic impairment is not expected to affect the systemic clearance of meropenem/ vaborbactam [17]. Because of reduced plasma clearance of meropenem and vaborbactam in patients with renal dysfunction [50], meropenem/vaborbactam dosage adjustments are required in patients with renal impairment (eGFR $<50 \mathrm{~mL} /$ $\mathrm{min} / 1.73 \mathrm{~m}^{2}$; Sect. 6). As renal impairment is more likely in the elderly, dosage adjustments in these patients should also be based on renal function [17].

Coadministration of valproic acid or divalproex sodium with carbapenems, such as meropenem, may reduce serum concentrations of valproic acid, resulting in increased risk of breakthrough seizures [17]. Consideration should be given to supplemental anticonvulsant therapy if administration of meropenem/vaborbactam is necessary [17]. Coadministration of probenecid with meropenem/vaborbactam is not recommended as probenecid competes with meropenem for active tubular secretion, leading to increased meropenem plasma concentrations [17]. During simulated Y-site administration, meropenem/vaborbactam was physically compatible with $83 \%$ of the 88 intravenous medications tested, including $82 \%$ of antibacterials tested (e.g. amikacin, gentamicin, tigecycline). Fifteen drugs (including albumin, ceftaroline, ciprofloxacin) were physically incompatible with meropenem/vaborbactam, with the most common incompatibility being change in turbidity [51].

$\begin{aligned} & \text { Table } 2 \\
& \text { Paborbactam. Mean values reported } \\
& \text { vabokinetic properties of meropenem and }\end{aligned}$
\begin{tabular}{llllll} 
Population $^{\mathrm{a}}$ & & $\begin{array}{l}\mathrm{C}_{\mathrm{max}} \\
(\mathrm{mg} / \mathrm{L})\end{array}$ & $\begin{array}{l}\mathrm{CL} \\
(\mathrm{L} / \mathrm{h})\end{array}$ & $\begin{array}{l}\mathrm{AUC}^{\mathrm{b}} \\
(\mathrm{mg} \cdot \mathrm{h} / \mathrm{L})\end{array}$ & $\begin{array}{l}\mathrm{t}_{1 / 2} \\
(\mathrm{~h})\end{array}$ \\
\hline Healthy adults $^{\mathrm{c}}$ & $\mathrm{MEM}$ & 43.4 & 15.1 & 414 & 1.22 \\
\hline & $\mathrm{VAB}$ & 55.6 & 10.9 & 588 & 1.68 \\
\hline Patients $^{\mathrm{c}}$ & $\mathrm{MEM}$ & 57.3 & 10.5 & 650 & 2.30 \\
\hline & $\mathrm{VAB}$ & 71.3 & 7.95 & 835 & 2.25
\end{tabular}

$A U C$ area under the concentration-time curve, $C L$ plasma clearance, $C_{\max }$ peak observed concentration, $M E M$ meropenem, $t_{1 / 2}$ elimination half-life, $V A B$ vaborbactam

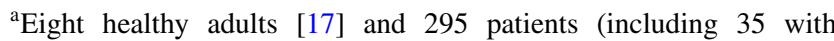
impaired renal function) [49] received approved dosages (Sect. 6)

${ }^{\mathrm{b}} \mathrm{AUC}_{24 \mathrm{~h}}$ (healthy adults) and $\mathrm{AUC}_{24 \mathrm{~h}}$ at steady state (patients)

${ }^{\mathrm{c}}$ Multiple-dose (7 days) pharmacokinetics (healthy adults) [17] and population pharmacokinetics (patients) [17, 49] 


\section{Therapeutic Efficacy of Meropenem/ Vaborbactam}

The randomized, double-blind, multinational, phase 3 TANGO I study evaluated the efficacy of meropenem/ vaborbactam versus piperacillin/tazobactam in adults with cUTI [52]. Eligible patients were $\geq 18$ years of age, had a bodyweight of $\leq 185 \mathrm{~kg}$, required intravenous antibacterials for $\geq 5$ days and had documented cUTI or acute pyelonephritis. Patients who required either an antibacterial in addition to meropenem/vaborbactam or an antifungal, those who had received an antibacterial within $48 \mathrm{~h}$ before randomization (except single-dose short-acting oral or intravenous antibacterial) or had an estimated creatinine clearance $\left(\mathrm{eCL}_{\mathrm{CR}}\right)$ of $<30 \mathrm{~mL} / \mathrm{min}$ were excluded from the study [52].

Patients received meropenem/vaborbactam (2/2 g via 3 -h intravenous infusion) or piperacillin/tazobactam (4.0/0.5 g via 30-min infusion) every $8 \mathrm{~h}$ for 10 days of total treatment (intravenous with or without oral therapy) [52]. Patients were stratified for randomization according to geographic region (North America, Europe, Asia Pacific and the rest of the world) and the type of infection (acute pyelonephritis, cUTI with removable focus and cUTI with nonremovable focus). In the meropenem/vaborbactam group, blinded dose adjustment was made for patients with $\mathrm{eCL}_{\mathrm{CR}}<50 \mathrm{~mL} /$ min; no dosage adjustment of piperacillin/tazobactam was required based on renal function. To complete 10 days of total treatment, patients who met prespecified criteria of improvement after 15 doses of intravenous treatment could be switched to oral levofloxacin (500 mg mg/day) [52].

Efficacy outcomes were assessed on day 3 of study treatment, at end of intravenous therapy (EOIVT), on the last day of total therapy (end of treatment; EOT), at the testof-cure (TOC) visit (i.e. EOT plus 7 days) and at the late follow-up visit (i.e. EOT plus 14 days) [52]. The efficacy analysis populations were: (1) modified intent-to-treat population (MITT), i.e. all patients who received $\geq 1$ dose of study drug, (2) modified-MITT (m-MITT) population, i.e., all patients in the MITT population with $\geq 1$ bacterial pathogen of $\geq 10^{5} \mathrm{CFU} / \mathrm{mL}$ in baseline urine culture or the same bacterial pathogen present in concurrent blood and urine cultures, and (3) the microbiological evaluable (ME) population, i.e. all m-MITT patients who had a clinical outcome and microbiological outcome at EOIVT; received $\geq 80$ and $\leq 120 \%$ of expected intravenous doses for completed treatment duration; missed 0 or 1 intravenous dose in the first $48 \mathrm{~h}$ and missed $\leq 2$ consecutive intravenous doses overall; received $\geq 6$ doses of study drug if classified as experiencing treatment failure on overall outcome; or received $\geq 9$ doses if classified as experiencing cure on overall outcome [52].

At baseline, the mean age of patients in the MITT population was $\approx 53$ years and patients in both the MITT and
m-MITT populations presented with a mean of 3.5 symptoms. In the MITT meropenem/vaborbactam $(n=272)$ and piperacillin/tazobactam $(n=273)$ groups, 59.2 and $59.0 \%$ of patients had acute pyelonephritis, 19.5 and $18.7 \%$ had cUTI with removable infection source, and 21.3 and $22.3 \%$ had cUTI with nonremovable infection source [52]. The common urinary pathogens in the m-MITT meropenem/vaborbactam $(n=192)$ and piperacillin/tazobactam $(n=182)$ groups were E. coli (65.1 and 64.3\%), K. pneumoniae (15.6 and 15.4\%), Enterococcus Faecalis (6.8 and 7.7\%), Proteus Mirabilis (3.1 and 6.6\%) and E. cloacae spp. complex (5.2 and 2.7\%). Of the urinary isolates tested at baseline in this population, almost all were susceptible to meropenem and $\approx 12 \%$ were resistant to piperacillin/tazobactam (FDA criteria) [52].

In the MITT population, the mean duration of intravenous and oral levofloxacin step-down therapy was 10.1 days in the meropenem/vaborbactam group and 9.9 days in the piperacillin/tazobactam group; the mean duration of intravenous therapy in both groups was 8.0 days [52]. Levofloxacin was used in $93.6 \%$ of meropenem/vaborbactam and $95.1 \%$ of piperacillin/tazobactam recipients; 9.9 and $8.2 \%$ of patients in the respective groups received levofloxacin despite having a levofloxacin-resistant pathogen at baseline (FDA criteria). Most patients in the meropenem/vaborbactam (91.5\%) and piperacillin/tazobactam groups $(86.1 \%)$ in the MITT population completed study treatment (intravenous plus oral) [52].

The primary efficacy endpoint was assessed using the FDA and European Medicines Agency (EMA) criteria.

- The FDA primary endpoint was the proportion of patients in the m-MITT population who achieved overall success at EOIVT visit. Overall success was the composite of clinical cure (complete resolution or significant improvement of baseline signs and symptoms of cUTI or acute pyelonephritis) and microbial eradication (baseline pathogens reduced to $<10^{4} \mathrm{CFU} / \mathrm{mL}$ urine).

- The EMA primary endpoint was the proportion of patients in the coprimary m-MITT and ME populations who achieved microbiological eradication (baseline pathogens reduced to $<10^{3} \mathrm{CFU} / \mathrm{mL}$ urine) at TOC visit.

Noninferiority was demonstrated if the lower limit of the two-sided $95 \%$ CI of the difference between the meropenem/ vaborbactam and piperacillin/tazobactam groups at EOIVT was greater than $-15 \%$ for overall success (FDA) and for microbial eradication at TOC in both the m-MITT and ME populations (EMA) [52]. If the FDA or EMA endpoints were noninferior, superiority of meropenem/vaborbactam over piperacillin/tazobactam was assessed using the $95 \% \mathrm{CI}$ to assess whether the lower bound of the 2-sided $95 \%$ CI was greater than 0 .

Meropenem/vaborbactam was noninferior to piperacillin/ tazobactam for the FDA primary endpoint of overall success 
(composite of clinical cure and microbial eradication) at EOIVT [98.4 vs. 94.0\%; between-group difference (BGD) $4.5 \%$; 95\% CI 0.7-9.1] and the EMA primary endpoint of microbial eradication in the m-MITT (66.7 vs. $57.7 \%$; BGD $9.0 \%$; $95 \% \mathrm{CI}-0.9$ to 18.7 ) and ME populations (66.3 vs. 60.4\%; BGD 5.9 (95\% CI -4.2 to 16.0) (all $p<0.001$ for noninferiority) [52]. Having established noninferiority, the superiority of meropenem/vaborbactam to piperacillin/tazobactam was shown for overall success $(p=0.01)$. Findings of the primary analysis were supported by a sensitivity analysis of overall success at EOIVT in patients who were cured, with noninferiority of meropenem/vaborbactam to piperacillin/tazobactam demonstrated (81.3 vs. $78.6 \% ; 2.7 \%$; $95 \%$ $\mathrm{CI}-5.5$ to 10.9 ).

For secondary endpoints, although the study was not powered to evaluate noninferiority of the two groups, noninferiority criteria were met for all key endpoints, including overall success at TOC (74.5 vs. $70.3 \%$; BGD $4.1 \%$; $95 \% \mathrm{CI}-4.9$ to 9.1 ), and overall success at EOIVT in patients with acute pyelonephritis (97.5 vs. $94.1 \%$; BGD $3.4 \%$; $95 \%$ CI -2.0 to 10.2 ), cUTI with removable infection source (100 vs. $92.1 \%$; BGD $7.9 \%$; $95 \% \mathrm{CI}-2.5$ to $20.9)$ and cUTI with non-removable infection source (100 vs. $95.3 \%$; BGD $4.7 \%$; $95 \%$ CI -5.1 to 15.6) [52]. Clinical cure rates with meropenem/vaborbactam at EOIVT (98.4 vs. $95.6 \%$; BGD $2.8 \%$; $95 \% \mathrm{CI}-0.7$ to 7.1$)$ and at TOC (90.6 vs. $86.3 \%$; BGD $4.4 \%$; $95 \%$ CI -2.2 to 11.1 ), as well as the proportion of patients with microbial eradication (FDA criteria) at EOIVT (97.9 vs. 82.3\%; BGD 5.6\%; 95\% CI 1.4 to 10.7 ) and TOC (68.8 vs. $62.1 \%$; BGD 6.7\%; $95 \% \mathrm{CI}-3.0$ to 16.2 ) were also noninferior to those with piperacillin/tazobactam ([52].

Microbial eradication rates at TOC in both groups $(68.8 \%$ in meropenem/vaborbactam and $62.1 \%$ in piperacillin/tazobactam recipients) were numerically lower than the rates at EOIVT (97.9 and 82.3\%), which largely accounted for the numerically lower overall success rates at TOC (74.5 and 70.3\%) than at EOIVT (98.4 and 94.0\%) [52]. These results may have been affected by the $\approx 10 \%$ of patients (distributed equally in both treatment groups) who received oral levofloxacin despite having levofloxacin-resistant microorganisms; however, clinical cure rates in both groups were high at TOC and EOIVT, indicating that most patients with microbiological recurrence or persistence had asymptomatic bacteriuria [52].

Overall success rates at EOIVT in subgroup analyses (e.g. based on age, race, geographic region) in the m-MITT population were generally consistent with the primary endpoint results, with the exception of outcomes in subgroups with small numbers of patients $(n<25)$ [52]. For example, in patients with bacteraemia at baseline, the overall success rates in meropenem/vaborbactam and piperacillin/tazobactam recipients were $83.3 \%$ (10/12 patients) and 100\%
(15/15) (between-group difference - 16.7\%; 95\% CI - 45.4 to $6.3 \%$ ); the two meropenem/vaborbactam recipients who did not achieve overall success with therapy had treatment failure because of treatment discontinuation due to an adverse event [52].

Overall success rates were also assessed according to underlying comorbidities (Charlson comorbidity score $\leq 2$ or $\geq 3 ; 0$ indicates no comorbidity) and severity of illness [systemic inflammatory response syndrome (SIRS) status yes/no] in patients receiving meropenem/vaborbactam or piperacillin/tazobactam [53]. At EOIVT, in the m-MITT population, overall success rates in both groups exceeded 90\% regardless of SIRS status or Charlson comorbidity score. At TOC, overall success rates were numerically higher in patients with SIRS (92.7\% in meropenem/vaborbactam and $72.1 \%$ in piperacillin/tazobactam groups) than in patients without SIRS (67.2 and 69.4\%), and among patients with SIRS, the rates were numerically higher in meropenem/ vaborbactam than piperacillin/tazobactam recipients (92.7 vs. $72.1 \%$ ). Patients with a Charlson comorbidity score of $\geq 3$ had numerically higher overall success rates at TOC in both treatment groups $(84.3 \%$ in meropenem/vaborbactam and $85.7 \%$ in piperacillin/tazobactam recipients) than in patients with a score of $\leq 2$ (66.0 and 59.0\%) [53].

In other m-MITT analyses, overall success rates at EOIVT and TOC were generally similar between meropenem/vaborbactam and piperacillin/tazobactam recipients regardless of renal function $\left(\mathrm{CL}_{\mathrm{CR}} \geq 50 \mathrm{~mL} / \mathrm{min}\right.$ or $\mathrm{CL}_{\mathrm{CR}}<50 \mathrm{~mL} / \mathrm{min}$ ) [54]. In addition, the clinical cure and microbial eradication rates at these timepoints were generally similar in the two groups regardless of baseline pathogen [32] and across a range of Enterobacteriaceae MICs [52]. In the $9.9 \%$ of patients in each group who were either in or were admitted to the intensive care unit (ICU) at the time of enrollment, the mean length of stay in the ICU did not differ significantly between patients in the meropenem/vaborbactam and piperacillin/tazobactam groups (9.3 vs. 11.1 days) [55].

\section{Tolerability of Meropenem/Vaborbactam}

Intravenous meropenem/vaborbactam was generally well tolerated in TANGO I in patients with cUTI, with a tolerability profile generally similar to that of intravenous piperacillin/ tazobactam [52]. In the meropenem/vaborbactam $(n=272)$ and piperacillin/tazobactam $(n=273)$ groups in the MITT population, treatment-emergent adverse events (AEs) occurred in 39.0 and $35.5 \%$ of patients, treatment-related AEs in 15.1 and $12.8 \%$ of patients, serious AEs in 4.0 and $4.4 \%$ of patients and life-threatening AEs (e.g. congestive heart failure, infusion-related reaction) in 1.1 and $0 \%$ of patients. Treatment-emergent AEs resulted in discontinuation of therapy in $2.6 \%$ of meropenem/vaborbactam and 
$5.1 \%$ of piperacillin/tazobactam recipients, and $1.1 \%$ of patients in each group withdrew from the study because of these AEs; there were two $(0.7 \%)$ deaths in each treatment group. The most common (incidence $>2 \%$ ) treatment emergent AEs in meropenem/vaborbactam recipients were headache ( 8.8 vs. $4.4 \%$ of piperacillin/tazobactam recipients), diarrhoea (3.3 vs. $4.4 \%$ ) and infusion-site phlebitis (2.2 vs. $0.7 \%$ ). Severe AEs occurred in $2.6 \%$ of meropenem/ vaborbactam and $4.8 \%$ of piperacillin/tazobactam recipients, with individual AEs (e.g. anaemia, increased levels of aspartate aminotransferase) occurring in $<1 \%$ of patients in either treatment group [52].

$\beta$-lactam antibacterials have been associated with serious and sometimes fatal hypersensitivity reactions and serious skin reactions; there have been reports of severe hypersensitivity reactions in patients with a history of penicillin hypersensitivity receiving another $\beta$-lactam antibacterial [17]. Thus, it is important to inquire about hypersensitivity to penicillin, cephalosporins, other $\beta$-lactam antibacterials and other allergens prior to meropenem/vaborbactam therapy. Meropenem has been associated with seizures and other adverse central nervous system (CNS) experiences, usually in patients with CNS disorders or bacterial meningitis and/ or compromised renal function. Only recommended dosages of meropenem/vaborbactam should be administered, and patients with known seizure disorders should continue receiving anticonvulsant therapy. Patients experiencing focal tremors, myoclonus or seizures should be evaluated neurologically, and may require anticonvulsant therapy (if not already instituted) and dosage adjustment or discontinuation of meropenem/vaborbactam [17].

As with almost all antibacterials, Clostridium difficileassociated diarrhoea (CDAD), ranging from mild to fatal colitis, has been reported with meropenem/vaborbactam [17]. As CDAD was reported to occur over a 2-month period after administration of antibacterials, it must be considered in all patients with diarrhoea after treatment with antibacterials. In the event of suspected or confirmed CDAD in meropenem/vaborbactam recipients, discontinuation of ongoing antibacterial treatment not directed against $C$. difficile may be required; fluids and electrolytes, protein supplement, antibacterials against $C$. difficile and surgical evaluation should be instituted as clinically indicated. Thrombocytopenia has been reported in patients with renal impairment who were treated with meropenem; however, no clinical bleeding was reported in these patients. Prolonged use of meropenem/ vaborbactam, as with other antibacterials, may lead to the overgrowth of nonsusceptible organisms; therefore repeated evaluation of patients is necessary, and appropriate measures should be taken if superinfection occurs during meropenem/ vaborbactam therapy [17].

\section{Dosage and Administration of Meropenem/Vaborbactam}

In the USA, meropenem/vaborbactam is indicated for the treatment of patients $\geq 18$ years of age with cUTI, including pyelonephritis, caused by the following susceptible organisms: E. coli, K. pneumoniae and E. cloacae [17]. To reduce the development of drug-resistant bacteria and maintain the efficacy of meropenem/vaborbactam, it should be used only to treat or prevent infections that are proven or strongly suspected to be caused by susceptible bacteria. The recommended dosage of meropenem/vaborbactam is $4 \mathrm{~g}$ (meropenem $2 \mathrm{~g}$ and vaborbactam $2 \mathrm{~g}$ ) administered every $8 \mathrm{~h}$ by intravenous 3 -h infusion in patients with an eGFR of $\geq 50 \mathrm{ml} / \mathrm{min} / 1.73 \mathrm{~m}^{2}$. The duration of treatment is $\leq 14$ days. In patients with impaired renal function, meropenem/vaborbactam dosage (administered as a 3-h infusion) should be reduced as follows [17]:

- $2 \mathrm{~g}$ (meropenem $1 \mathrm{~g}$ and vaborbactam $1 \mathrm{~g}$ ) every $8 \mathrm{~h}$ in patients with eGFR of 30-49 $\mathrm{ml} / \mathrm{min} / 1.73 \mathrm{~m}^{2}$

- $2 \mathrm{~g}$ (meropenem $1 \mathrm{~g}$ and vaborbactam $1 \mathrm{~g}$ ) every $12 \mathrm{~h}$ in patients with eGFR of $15-29 \mathrm{ml} / \mathrm{min} / 1.73 \mathrm{~m}^{2}$

- $1 \mathrm{~g}$ (meropenem $0.5 \mathrm{~g}$ and vaborbactam $0.5 \mathrm{~g}$ ) every $12 \mathrm{~h}$ in patients with eGFR of $<15 \mathrm{ml} / \mathrm{min} / 1.73 \mathrm{~m}^{2}$

As meropenem and vaborbactam are removed by haemodialysis, patients maintained on haemodialysis should be administered meropenem/vaborbactam after a haemodialysis session [17]. In patients with changing renal function, serum creatinine concentrations and eGFR should be monitored at least once a day and meropenem/vaborbactam dosages adjusted accordingly. Local prescribing information should be consulted for further information, including dosage and administration details, contraindications, warnings and precautions.

\section{Current Status of Meropenem/ Vaborbactam for The Treatment of cUTIs}

Antibacterials have been the mainstay of treatment of cUTIs, with the choice of therapy based on factors such as patient tolerance, severity of underlying urological abnormality, previous antibacterial therapy, urine culture reports, spectrum of local pathogens and local antimicrobial resistance patterns $[3,4,8]$. Initial empirical therapy should include antibacterials with broad-spectrum activity (e.g. third-generation cephalosporins, aminoglycosides or carbapenems), based on the spectrum of local pathogens [3-5]. However, because of the emergence and spread of antibacterial resistance in 
common UTI pathogens $[2,4]$, there is a need for new highly effective antibacterials.

Intravenous meropenem/vaborbactam is the first carbapenem/BLI combination approved in the USA for use in patients with cUTI, including pyelonephritis [17]. When combined with meropenem, vaborbactam restores the activity of meropenem against $\beta$-lactamase-producing Enterobacteriaceae (Sect. 2). Meropenem/vaborbactam demonstrated excellent in vitro activity against Gram-negative clinical isolates, including KPC- and ESBL-producing Enterobacteriaceae and MDR Enterobacteriaceae (Sect. 2). In the clinical setting, meropenem/vaborbactam was no less effective than intravenous piperacillin/tazobactam in terms of the composite endpoint of overall success (FDA primary endpoint) and microbial eradication (EMA primary endpoint) in the pivotal, phase 3, noninferiority TANGO I trial in patients with cUTI (Sect. 4). In subsequent superiority testing, meropenem/vaborbactam was superior to piperacillin/tazobactam in terms of overall success (Sect. 4). Meropenem/vaborbactam was generally well tolerated, with a tolerability profile generally similar to that of piperacillin/tazobactam (Sect. 5).

With regard to the comparator, although piperacillin is approved for the treatment of UTIs in the USA, piperacillin/tazobactam is not approved for patients with cUTI (it is approved for cUTIs in other countries) [4]. However, because of its antibacterial spectrum, time-dependent bacterial killing, pharmacokinetic properties, three times daily dosing and achievement of high urinary concentrations, the FDA approved its use as a comparator in TANGO I [4]. The dosage of piperacillin/tazobactam selected for the study (4.0/0.5 g every $8 \mathrm{~h}$ ) was also approved by the FDA and differed from the usual daily dose of 3.0/0.375 g every $6 \mathrm{~h}$ in patients with an eGFR of $>40 \mathrm{~mL} / \mathrm{min}$ [4].

It should be noted that while patients enrolled in TANGO I were symptomatic and had pyuria, urine cultures with pathogen growth of $>10^{3} \mathrm{CFU} / \mathrm{mL}$ and significant comorbidities, $<30 \%$ of patients met the criteria of SIRS and only $3 \%$ of patients were enrolled in the USA [52]. It is unclear if all the patients would have met the criteria of hospitalization in the USA [52]. TANGO I also did not assess the efficacy of meropenem/vaborbactam for the treatment of infections caused by CRE, as it would have been unethical to administer the comparator piperacillin/tazobactam in these patients [52]. A second phase 3 study, TANGO II (NCT02168946) compared the efficacy of meropenem/vaborbactam with best available therapy in patients with cUTI, acute pyelonephritis, hospital-acquired or ventilator-associated bacterial pneumonia, bacteraemia or complicated intra-abdominal infection due to known or suspected CRE. Recently presented data showed that meropenem/vaborbactam was associated with significantly higher cure rates relative to best available therapy [56]. Fully published results from TANGO II are awaited with interest and it should be noted that meropenem/vaborbactam is currently not approved for use in these patients. Additional studies are needed to determine which patient groups may be more likely to benefit from meropenem/vaborbactam therapy [52].

To conclude, meropenem/vaborbactam is a broad-spectrum antibacterial developed for use against serious Gram-negative infections, including those caused by KPC-producing Enterobacteriaceae. Meropenem/vaborbactam was effective and generally well tolerated in the well-designed TANGO I study in patients with cUTI, and is the first carbapenem/BLI combination to be approved by the FDA in this indication. Although fully published results regarding its efficacy in the treatment of infections caused by CRE and additional data identifying groups of patients who may be more likely to benefit from therapy are awaited, current evidence indicates that meropenem/vaborbactam is a useful treatment option for patients with cUTIs.

\section{Data Selection Drug Name: 128 records identified}

Duplicates removed

Excluded during initial screening (e.g. press releases; news reports; not relevant drug/indication; preclinical study; reviews; case reports; not randomized trial)

Excluded during writing (e.g. reviews; duplicate data; small patient number; nonrandomized/phase I/II trials)

Cited efficacy/tolerability articles

Cited articles not efficacy/tolerability

Search Strategy: EMBASE, MEDLINE and PubMed from 1946 to present. Clinical trial registries/databases and websites were also searched for relevant data. Key words were Vabomere, meropenem, RPX-2014, vaborbactam, RPX-7009. Records were limited to those in English language. Searches last updated 10 August 2018

Acknowledgements During the peer review process, the manufacturer of meropenem/vaborbactam was also offered an opportunity to review this article. Changes resulting from comments received were made on the basis of scientific and editorial merit.

\section{Compliance with Ethical Standards}

Funding The preparation of this review was not supported by any external funding.

Conflict of interest Sohita Dhillon is a salaried employee of Adis/ Springer, is responsible for the article content and declares no relevant conflicts of interest.

Open Access This article is distributed under the terms of the Creative Commons Attribution-NonCommercial 4.0 International License (http://creativecommons.org/licenses/by-nc/4.0/), which permits any noncommercial use, duplication, adaptation, distribution and reproduction in any medium or format, as long as you give appropriate credit to the original author(s) and the source, provide a link to the Creative Commons license and indicate if changes were made. 


\section{References}

1. Vallejo-Torres L, Pujol M, Shaw E, et al. Cost of hospitalised patients due to complicated urinary tract infections: a retrospective observational study in countries with high prevalence of multidrug-resistant Gram-negative bacteria: the COMBACTEMAGNET, RESCUING study. BMJ Open. 2018;8(4):e020251.

2. Flores-Mireles AL, Walker JN, Caparon M, et al. Urinary tract infections: epidemiology, mechanisms of infection and treatment options. Nat Rev Microbiol. 2015;13(5):269-84.

3. European Association of Urology. Guidelines on urological infections. 2015. https://uroweb.org/wp-content/uploads/19Urological-infections_LR2.pdf. Accessed 30 July 2018.

4. US Center for Drug Evaluation and Research. Clinical review(s). 2016. https://www.accessdata.fda.gov/drugsatfda _docs/nda/2017/209776Orig1s000MedR.pdf. Accessed 30 July 2018.

5. Ma AH, Hughes GJ. Updates in management of complicated urinary tract infections: a focus on multidrug-resistant organisms. Am J Ther. 2018;25:e53-66.

6. Codjoe FS, Donkor ES. Carbapenem resistance: a review. Med Sci. 2017;6(1):1

7. Papp-Wallace KM, Endimiani A, Taracila MA, et al. Carbapenems: past, present, and future. Antimicrob Agents Chemother. 2011;55(11):4943-60

8. Bader MS, Loeb M, Brooks AA. An update on the management of urinary tract infections in the era of antimicrobial resistance. Postgrad Med. 2017;129(2):242-58.

9. US Centers for Disease Control and Prevention. Facility guidance for control of carbapenem-resistant Enterobacteriaceae (CRE). 2015. https://www.cdc.gov/hai/pdfs/cre/CRE-guidance-508.pdf. Accessed 30 July 2018.

10. European Center for Disease Control and Prevention. Carbapenem-resistant Enterobacteriaceae. 2016. https://ecdc.europa.eu/ sites/portal/files/media/en/publications/Publications/carbapenem -resistant-enterobacteriaceae-risk-assessment-april-2016.pdf. Accessed 30 July 2018.

11. US Centers for Disease Control and Prevention. Antibiotic resistance threats in the United States, 2013. 2013. https://www.cdc. gov/drugresistance/pdf/ar-threats-2013-508.pdf. Accessed 30 July 2018.

12. World Health Organization. WHO publishes list of bacteria for which new antibiotics are urgently needed. 2017. http://www.who. int/news-room/detail/27-02-2017-who-publishes-list-of-bacteriafor-which-new-antibiotics-are-urgently-needed. Accessed 30 July 2018.

13. van Duin D, Doi Y. The global epidemiology of carbapenemaseproducing Enterobacteriaceae. Virulence. 2017;8(4):460-9.

14. Ye Y, Xu L, Han Y, et al. Mechanism for carbapenem resistance of clinical Enterobacteriaceae isolates. Exp Ther Med. 2018;15(1):1143-9.

15. Hecker SJ, Reddy KR, Totrov M, et al. Discovery of a cyclic boronic acid beta-lactamase inhibitor (RPX7009) with utility vs class A serine carbapenemases. J Med Chem. 2015;58(9):3682-92.

16. Lomovskaya O, Sun D, Rubio-Aparicio D, et al. Vaborbactam: spectrum of beta-lactamase inhibition and impact of resistance mechanisms on activity in Enterobacteriaceae. Antimicrob Agents Chemother. 2017;61(11):e01443-17.

17. The Medicines Company. VABOMERE ${ }^{\mathrm{TM}}$ (meropenem and vaborbactam): US prescribing Information. 2018. https://www. accessdata.fda.gov/drugsatfda_docs/label/2018/209776s001lbl. pdf. Accessed 30 July 2018.

18. Zhanel GG, Lawrence $\mathrm{CK}$, Adam H, et al. Imipenemrelebactam and meropenem-vaborbactam: two novel carbapenem-beta-lactamase inhibitor combinations. Drugs. 2018;78(1):65-98.

19. Cho JC, Zmarlicka MT, Shaeer KM, et al. Meropenem/vaborbactam, the first carbapenem/beta-lactamase inhibitor combination. Ann Pharmacother. 2018;52(8):769-79.

20. Lomovskaya O, Tsivkovski R. Vaborbactam (RPX7009) plus meropenem is active against the newly discovered BKC-1 and FR-1 carbapenemases [abstract no. P1289]. In: 26th ECCMID. 2016.

21. Castanheira M, Rhomberg PR, Flamm RK, et al. Effect of the $\beta$-lactamase inhibitor vaborbactam combined with meropenem against serine carbapenemase-producing Enterobacteriaceae. Antimicrob Agents Chemother. 2016;60(9):5454-8.

22. US Center for Drug Evaluation and Research. Clinical microbilology/virology review(s). 2017. https://www.accessdata.fda. gov/drugsatfda_docs/nda/2017/209776Orig 1s000MicroR.pdf. Accessed 30 July 2018.

23. Tsivkovski R, Griffith DC, Hecker S, et al. Biochemical characterization of the beta-lactamase inhibitor RPX7009 [abstract no. F-849 plus poster]. In: 52nd ICAAC. 2012.

24. Lapuebla A, Abdallah M, Olafisoye O, et al. Activity of meropenem combined with RPX7009, a novel $\beta$-lactamase inhibitor, against Gram-negative clinical isolates in New York city. Antimicrob Agents Chemother. 2015;59(8):4856-60.

25. Castanheira M, Huband MD, Mendes RE, et al. Meropenemvaborbactam tested against contemporary Gram-negative isolates collected worldwide during 2014, including carbapenemresistant, KPC-producing, multidrug-resistant, and extensively drug-resistant Enterobacteriaceae. Antimicrob Agents Chemother. 2017;61(9):e00567-17.

26. Castanheira M, Huband MD, Flamm RK, et al. Meropenemvaborbactam (MER-VAB) tested against contemporary Enterobacteriaceae isolates from USA hospitals [abstract no. 452 plus poster]. In: ASM microbe. 2016.

27. Castanheira M, Mendes RE, Flamm RK, et al. Activity of meropenem/RPX7009 and comparator agents tested against contemporary Enterobacteriaceae isolates collected from bloodstream infections in USA hospitals [abstract no. C-152 plus poster]. In: 55th ICAAC. 2015.

28. Pfaller MA, Huband MD, Mendes RE, et al. In vitro activity of meropenem-vaborbactam and characterization of carbapenem resistance mechanisms among carbapenem-resistant Enterobacteriaceae from the 2015 meropenem-vaborbactam surveillance program. Int J Antimicrob Agents. 2018;52(2):144-50.

29. Huband MD, Flamm RK, Rhomberg PR, et al. In vitro antibacterial activity of meropenem/RPX7009, (a carbapenem/ $\beta$ lactamase inhibitor combination) against contemporary Enterobacteriaceae (ENT) isolated from intra-abdominal (IAI) and urinary tract infections (UTI) in the United States [abstract no. 781 plus poster]. Open Forum. Infect Dis. 2015;2(Suppl 1):781.

30. Castanheira M, Duncan LR, Deshpande L, et al. Meropenemvaborbactam activity against Enterobacteriaceae isolates collected during 2014-2015 from European countries [abstract no. P128]. In: 27th ECCMID congress. 2017.

31. Castanheira M, Arends SJR, Deshpande LM, et al. Activity of meropenem-vaborbactam against Enterobacteriaceae isolates collected during 2016 [abstract no. P1043 plus poster]. In: 28th ECCMID congress. 2018.

32. Walsh TJ, Bhowmick T, Darouiche R, et al. Per pathogen outcomes of meropenem-vaborbactam (M-V) versus piperacillintazobactam (P-T) in the treatment of adults with complicated urinary tract infections (cUTI), including acute pyelonephritis (AP), in TANGO 1, a phase 3 randomized, double-blind, double-dummy trial [abstract no. P1290 plus poster]. In: 27th ECCMID congress. 2017. 
33. Hackel MA, Lomovskaya O, Dudley MN, et al. In vitro activity of meropenem-vaborbactam against clinical isolates of KPCpositive Enterobacteriaceae. Antimicrob Agents Chemother. 2018;62(1):1-10.

34. Yang Q, Zhang G, Xu Y. In vitro activity of meropenem combined with RPX7009 against Enterobacteriaceae producing KPC-type carbapenemases in China [abstract no. P1285]. In: 27th ECCMID congress. 2017.

35. Perez F, El Chakhtoura NG, Papp-Wallace KM, et al. Treatment options for infections caused by carbapenem-resistant Enterobacteriaceae: can we apply "precision medicine" to antimicrobial chemotherapy? Expert Opin Pharmacother. 2016;17(6):761-81.

36. Weiss WJ, Pulse ME, Nguyen P, et al. Activity of meropenemvaborbactam against carbapenem-resistant Enterobacteriaceae in a murine model of pyelonephritis. Antimicrob Agents Chemother. 2018;62(1):e01439-17.

37. Sabet M, Tarazi Z, Nolan T, et al. Activity of meropenem-vaborbactam in mouse models of infection due to KPC-producing carbapenem-resistant Enterobacteriaceae (CRE). Antimicrob Agents Chemother. 2017;62(1):e01446-17.

38. Castanheira M, Mendes RE, Duncan LR, et al. Activity of meropenem-vaborbactam against Enterobacteriaceae isolates carrying blaKPC collected worldwide [abstract no. 1234 plus poster]. Open Forum. Infect Dis. 2017;4(Suppl. 1):S377.

39. Giddins MJ, Macesic N, Annavajhala MK, et al. Successive emergence of ceftazidime-avibactam resistance through distinct genomic adaptations in blaKPC-2-harboring Klebsiella pneumoniae sequence type 307 isolates. Antimicrob Agents Chemother. 2018;62(3):e02101-17.

40. Shields RK, Chen L, Cheng S, et al. Emergence of ceftazidimeavibactam resistance due to plasmid-borne blaKPC-3 mutations during treatment of carbapenem-resistant Klebsiella pneumoniae infections. Antimicrob Agents Chemother. 2017;61(3):e02097-16.

41. Humphries RM, Yang S, Hemarajata P, et al. First report of ceftazidime-avibactam resistance in a KPC-3-expressing Klebsiella pneumoniae isolate. Antimicrob Agents Chemother. 2015;59(10):6605-7.

42. Lomovskaya $\mathrm{O}$, Tsivkovski R. Vaborbactam (VAB) is not affected by KPC-2 and KPC-3 variants containing Asp179Tyr amino acid substitution that are resistant to ceftazidime (CAZ) potentiation with avibactam [abstract no. 95E plus poster]. In: MAD-ID annual meeting. 2018

43. Tsivkovski R, Totrov M, Lomovskaya O. Inhibition of KPC-2 by vaborbactam (VAB; formerly RPX7009) does not involve Ser130 (S130) that is important for its inhibition by avibactam (AVI) [abstract no. 373]. In: ASM microbe. 2016.

44. Sun D, Rubio-Aparicio D, Nelson K, et al. Meropenem-vaborbactam: resistance selection, prevention, and molecular mechanisms in mutants of KPC-producing Klebsiella pneumoniae. Antimicrob Agents Chemother. 2017;61(12):e01702-17.

45. Sabet M, Tarazi Z, Rubio-Aparicio D, et al. Activity of simulated human dosage regimens of meropenem and vaborbactam against carbapenem-resistant Enterobacteriaceae in an in vitro hollow fiber model. Antimicrob Agents Chemother. 2018;62(2):e01969-17.

46. US Center for Drug Evaluation and Research. Clinical pharmacology and biopharmaceutics review(s). 2016. https://www.acces sdata.fda.gov/drugsatfda_docs/nda/2017/209776Orig1s000ClinP harmR.pdf. Accessed 30 July 2018.

47. Bhavnani SM, Trang M, Griffith DC, et al. Meropenem-vaborbactam pharmacokinetic-pharmacodynamic (PK-PD) target attainment analyses as support for dose selection in patients with normal renal function and varying degrees of renal impairment [abstract no. 1852 plus poster]. Open Forum. Infect Dis. 2017;4(Suppl. 1):S530-1.

48. Griffith D, Sabet M, Tarazi Z, et al. Pharmacodynamics of vaborbactam when administered in combination with meropenem [abstract no. Sunday-194]. In: ASM microbe. 2017.

49. Trang M, Griffith DC, Bhavnani SM, et al. Population pharmacokinetics (PPK) of mereopenem and vaborbactam in healthy volunteers and infected patients [abstract no. SUNDAY-192]. In: ASM microbe. 2017.

50. Rubino CM, Bhavnani SM, Loutit JS, et al. Single-dose pharmacokinetics and safety of meropenem-vaborbactam in subjects with chronic renal impairment. Antimicrob Agents Chemother. 2018;62(3):1-10.

51. Kidd JM, Avery LM, Asempa TE, et al. Physical compatibility of meropenem and vaborbactam with select intravenous drugs during simulated Y-site administration. Clin Ther. 2018;40(2):261-9.

52. Kaye KS, Bhowmick T, Metallidis S, et al. Effect of meropenem-vaborbactam vs piperacillin-tazobactam on clinical cure or improvement and microbial eradication in complicated urinary tract infection: the TANGO I randomized clinical trial. JAMA. 2018;319(8):788-99.

53. Shorr A, Loutit J, Dudley M, et al. Meropenem-vaborbactam $(\mathrm{M}-\mathrm{V})$ : clinical outcomes by comorbidity severity in TANGO 1 [abstract no. 306 plus poster]. In: ASM microbe. 2017.

54. Mathers A, Hope W, Kaye KS, et al. Meropenem-vaborbactam: outcomes in subjects with renal impairment in phase 3 studies TANGO I and II [abstract no. 1879 plus poster]. Open Forum. Infect Dis. 2017;4(Suppl. 1):S542.

55. Shorr A, Dufour S, Loutit J, et al. Hospital and ICU length of stay (LOS) with meropenem-vaborbactam (M-V) versus piperacillintazobactam (P-T) in complicated urinary tract infections (cUTI) including acute pyelonephritis (AP) in TANGO 1 [abstract no. 94E plus poster]. In: MAD-ID annual meeting. 2018.

56. Kaye KS, Vazquez J, Mathers A, et al. Clinical outcomes of serious infections due to carbapenem-resistant Enterobacteriaceae (CRE) in TANGO II, a phase 3, randomized, multinational, openlabel trial of meropenem-vaborbactam $(\mathrm{M}-\mathrm{V})$ vs. best available therapy (BAT) [abstract no. 1862 plus poster]. Open Forum Infect Dis. 2017;4(Suppl. 1):S534-5. 